DE L T A U N I V E R I T Y
SCIENTIFIC JOURNAL
Journal home page: www.deltauniv.edu.eg/dusj

\title{
Tunable Polarization Rotator Based on SOI Platform for Optical Communications Systems
}

Ahmed El-Sayed Abd-Elkader, ${ }^{1}$, Mohamed Farhat O. Hameed, 3,4,5, Nihal F.F. Areed ${ }^{2,4}$, Hossam El-Din Mostafa $^{2}$, and Salah S. A. Obayya ${ }^{2,4}$

${ }^{1}$ Delta University for Science \& Technology, Communications and Computer Engineering Department, Faculty of Engineering, International Costal Road, Gamasa, Dakahlia, Egypt.

${ }^{2}$ Mansoura University, Department of Electronics and Communication Engineering, Faculty of Engineering, Mansoura 35516, Egypt.

${ }^{3}$ Mansoura University, Department of Mathematics and Engineering Physics, Faculty of Engineering, Mansoura 35516, Egypt.

${ }^{4}$ Centre for Photonics and Smart Materials, Zewail City for Science and Technology, October Gardens, $6^{\text {th }}$ of October City, Giza, Egypt.

${ }^{5}$ Nanotechnology Engineering Program, Zewail City for Science and Technology, October Gardens, $6^{\text {th }}$ of October City, Giza, Egypt.

\section{Abstract}

A compact polarization rotator (PR) based on silicon on insulator (SOI) platform with nematic liquid crystal (NLC) is introduced. The NLCs are anisotropic materials which can be used to improve the modal hybridness of the hybrid modes. As a result, a polarization rotation can be achieved over a short propagation distance. The proposed PR is analyzed using the full-vectorial finite-difference method and the full-vectorial finite-difference beam propagation method. The numerical results reveal that at the operating wavelength of $1.55 \mu \mathrm{m}$, the PR has a compact device length of $11.67 \mu \mathrm{m}$ with $15.5 \mathrm{~dB}$ extinction ratio (ER) for both polarization conversions. The insertion losses of the reported PR are equal to $0.29 \mathrm{~dB}$ and $0.59 \mathrm{~dB}$ for TE to TM and for TM to TE conversions, respectively. Moreover, it offers a broad bandwidth of $(860-1780 \mathrm{~nm})$ for TE to TM and $(890-1790 \mathrm{~nm})$ for TM to TE with an ER better than $10 \mathrm{~dB}$. The fabrication tolerances and influences on the performance of the PR for the different structure geometrical parameters and operating wavelength are also investigated.

Index Terms-Polarization rotators, Polarization splitters, Nematic liquid crystal (NLC), Beam propagation method (BPM).

\section{Introduction}

As information exchange demands are exploding in today's communications, developing optical communications systems with increasing transmission capacities became a strong necessity. Optical communications systems rely heavily on the integration of different optical devices such as, detectors, modulators, and multiplexers. The performance of such devices is, unfortunately, usually dependent on the polarization state. Silicon photonics (1), (2) has emerged as a promising platform for building large scale photonic integrated circuits for optical communication systems due to the availability of the low cost complementary metal-oxide semiconductor processing technology. Silicon-oninsulator platform is the main platform for silicon photonics due to its high index contrast which enables compact photonic devices. However, the high index contrast leads to a considerable optical birefringence and highly polarization dependent devices. In this context, polarization handling devices (3), (4) such as polarization splitters and polarization rotators can be used to obtain polarization transparent PICs

Several approaches to implement polarization rotator based on SOI platform have been reported. In this regard, a compact PR based on asymmetric

Delta University for Science and Technology

Coastal International Road, Mansoura, Gamasa City, Dakahlia, Egypt

E-mail: dusj@deltauniv.edu.eg

Journal homepage: www.deltauniv.edu.eg/dusj 
periodic loaded waveguide has been introduced by Sun et al. ${ }^{(5)}$. At the wavelength of $1.525 \mu \mathrm{m}$, such a design has $15.78 \mu \mathrm{m}$ device length with $11.8 \mathrm{~dB}$ extinction ratio (ER). However, the structure was very sensitive to fabrication errors and narrow bandwidth ${ }^{(5)}$. Moreover, single section PRs ${ }^{(6)-(8)}$ with slanted sidewall were introduced with a complex fabrication process including wet and dry etching techniques. To overcome the problem of complex fabrication process, Wang et al ${ }^{\left({ }^{(9)}\right.}$ have proposed a PR based on $\mathrm{Si}$ nanowire with asymmetrical cross section through vertical sidewall and etched corner of the $\mathrm{Si}$ nanowire (one-step etched). Such structure has $22.1 \mu \mathrm{m}$ conversion length and broad bandwidth of 120 $\mathrm{nm}$. However, the fabrication tolerance was very small with high sensitivity to small fabrication errors. In addition, PRs based on directional coupling (10), (11) have been reported. Soudi et al. (11) have proposed a design of directional coupling based PR that contains two simple silicon nanowires with unequal widths. This design has a shorter device length of $52.8 \mu \mathrm{m}$ at $1.55 \mu \mathrm{m}$ wavelength with better maximum power coupling efficiency of $99 \%$ and $20 \mathrm{~dB}$ ER. However, directional coupler based PRs suffer from their relatively long device lengths. Further, several surface plasmon polariton (SPP) based PRs (12-14) have been implemented. Such technique is utilized to reduce the conversion length of an optical PR built on SOI waveguide. In this regard, an ultracompact SPP based PR with $3 \mu \mathrm{m}$ device length and ER of $11 \mathrm{~dB}$ has been reported ${ }^{(12)}$. However it suffers from high loss of $12 \mathrm{~dB}$ due to the metal existence. It is concluded that designing a PR which compromises between short device length, good ER, high PCE, low losses, large bandwidth with simple design is still an issue.

In this paper, a novel design of a compact PR based on SOI platform is introduced. The suggested design relies on a silicon wire inside a groove in the $\mathrm{SiO}_{2}$ substrate. The silicon wire is surrounded by nematic liquid crystals (NLCs). The anisotropic property of the NLCs ${ }^{(15)}$ leads to a high modal hybridness which improves the exchange of power between the hybrid modes. Optimizing the structural dimensions and the rotation angle of the NLC director leads to maximum modal hybridness, where the optical axis has an angle of $45^{\circ}$ with respect to the horizontal and vertical axes, can be obtained. Hence, maximum polarization conversion efficiency can be achieved over a short propagation distance. At a wavelength of $1.55 \mu \mathrm{m}$, the proposed design has a compact device length of $11.67 \mu \mathrm{m}$ with $15.5 \mathrm{~dB}$ ER for both polarization conversions. The IL is equal to $0.29 \mathrm{~dB}$ and 0.59 $\mathrm{dB}$ for TE to TM and for TM to TE conversions, respectively. Moreover, it offers a broad bandwidth of $(860-1780 \mathrm{~nm})$ for TE to TM mode and $(890-1790 \mathrm{~nm})$ for TM to TE mode to have an ER better than $10 \mathrm{~dB}$. In addition, the proposed PR is easy for fabrication and has a stable performance with variations in the operating wavelength and unavoidable fabrication errors. In order to study the characteristics of the proposed PR, the use of a full-vectorial mode solver is necessary. In this investigation, the fullvectorial finite difference method (FVFDM) with perfect matched layer boundary conditions ${ }^{(16)}$ is used to calculate the full vectorial quasi-transverse electric (TE) and transverse magnetic (TM) modes for the PR waveguide. In addition, the propagation along the longitudinal direction is studied using the full vectorial finite difference beam propagation method (FVFD-BPM) ${ }^{(17) .}$

This paper is organized as follows. Following Section I, the numerical methods are briefly described in Section II. The design and the obtained simulation results are presented in Section III. Finally, conclusions are drawn.

\section{Numerical Approaches}

In order to efficiently analyze the operation of the PRs, the use of full vectorial numerical approaches is inevitable. In this study, the FVFDM ${ }^{(16)}$ with perfect matched layer boundary conditions ${ }^{(18)}$ is used to accurately calculate the full vectorial quasi-TE and quasi-TM modes for the input and PR waveguides. The grid sizes of $\Delta x$ and $\Delta y$ in the transverse directions are taken as $0.01 \mu \mathrm{m}$ through all simulations.

To study the propagation along the longitudinal direction of a PR waveguide section, beam propagation methods (BPM) ${ }^{(17),(19)}$ are strongly required. In the last two decades, the BPM has been widely used for the simulation of wave propagation in different photonic devices. A fullvectorial approach is particularly necessary to calculate the power conversion between the two

Delta University for Science and Technology

Coastal International Road, Mansoura, Gamasa City, Dakahlia, Egypt

E-mail: dusj@deltauniv.edu.eg

Journal homepage: www.deltauniv.edu.eg/dusj 
polarization states in the optical guided wave devices. Due to its numerical efficiency and versatility, some full-vectorial BPM algorithms have been formulated based on the finite element method (FEM) ${ }^{(19) .}$ In addition, many full-vectorial BPM approaches based on the popular finite difference method (FDM) have been reported (17). In this study, the FVFD-BPM (17) is applied to study the polarization conversion based on the proposed structure. Through all simulations, the grid sizes are taken as $\Delta x=0.01 \mu \mathrm{m}, \Delta y=0.01$ $\mu \mathrm{m}$, and $\Delta z=0.01 \mu \mathrm{m}$ in the $\mathrm{x}, \mathrm{y}$, and $\mathrm{z}$ directions, respectively. In (19), obayya et al. have explained that for $\Delta z \leq 1 \mu \mathrm{m}$, the variation of the longitudinal step size does not have much influence on the accuracy of the scheme, and no instability was observed. In addition, $\alpha$ is taken within the range, $0.5 \leq \alpha \leq 1$, at which the FVFDBPM (17) is unconditionally stable. The $\alpha$ parameter is responsible for controlling the scheme which is used to solve the finite difference equations. Moreover, the reference index $n_{0}$ which is used to satisfy the slowly varying envelope approximation of the FVFD-BPM ${ }^{(17)}$ is taken as the effective index of the fundamental mode launched at the input waveguide.

Design And Numerical Results

The cross sections of the input/output and PR waveguides are shown in Fig. 1(a) and (b), respectively. The PR waveguide consists of a silicon wire of equal height $h_{s}$ and width $w_{s}$, which is positioned inside a groove in the $\mathrm{SiO}_{2}$ substrate and fixed by a strip of width $w_{b}$. The upper cladding and the regions in the groove surrounding the silicon wire are infiltrated with a NLC of type E7. The input and output waveguides have the same structure as the PR waveguide, while, the NLC is replaced by $\mathrm{SiO}_{2}$. The NLCs used in the proposed structure are anisotropic materials consisting of rod-like molecules, which are characterized by ordinary index $n_{o}$ and extraordinary index $n_{e}$. In addition, the direction of the preferred orientation of the NLCs, as shown in Fig. 1(d) is described by its director, which is a unit vector $n$ along the direction of the average orientation of the molecules. The orientation of the NLC director can be effectively controlled by applying a static electric field, where the NLC molecules tend to align their axis according to the applied field ${ }^{(20)}$.

The ordinary $n_{o}$ and extraordinary $n_{e}$ indices of the E7 material can be calculated using Cauchy models (21). In the proposed design, the relative permittivity tensor $\varepsilon_{r}$ is expressed as (22)

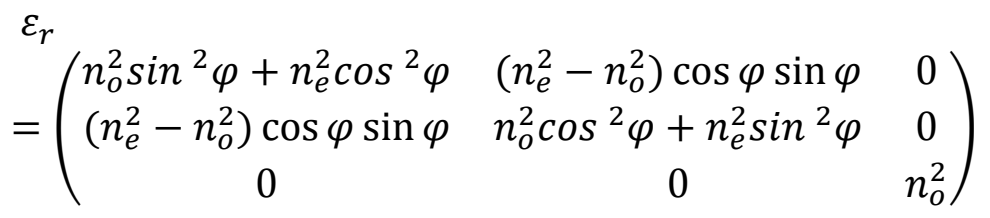

where $\varphi$ is the rotation angle of the NLC director with respect to the $\mathrm{X}$ - axis, as shown in the inset of Fig. 1. By optimizing the rotation angle, the optical axes of the fundamental quasi-TE and quasi-TM modes will have an angle of nearly $45^{\circ}$ with respect to the horizontal and vertical axes of the PR waveguide. Therefore, the dominant and nondominant field components of the two polarized modes will have nearly equal amplitudes. Accordingly, when a quasi-TE (TM) polarized mode obtained from the input waveguide, with little hybridization, is launched directly into the proposed PR, the input power excites two hybrid modes along the PR waveguide. As these two modes propagate through the

PR

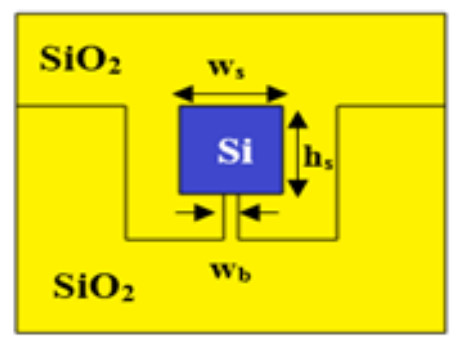

(a)

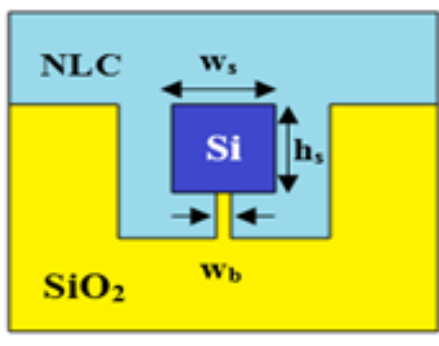

(b)

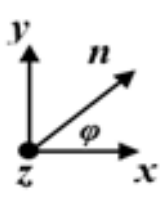

Delta University for Science and Technology

Coastal International Road, Mansoura, Gamasa City, Dakahlia, Egypt

E-mail: dusj@deltauniv.edu.eg

Journal homepage: www.deltauniv.edu.eg/dusj 
Fig. 1. (a) The cross-sections of the input/output waveguides, (b) the crosssection of the proposed PR waveguide. The section, they became out of phase at a distance equals to $L_{\pi}$, and then an orthogonal mode quasiTM (TE) is obtained.

The conversion length, $L_{C}$, or the half-beat length, $L_{\pi}$, is defined as the minimum longitudinal distance at which maximum polarization conversion occurs, and can be calculated by

$$
L_{C}=\pi /\left(\beta_{T E}-\beta_{T M}\right)
$$

where $\beta_{T E}$ and $\beta_{T M}$ are the propagation constants of the quasi-TE and quasi-TM modes, respectively. It should be noted that in order to reverse the polarization state at the output port of the PR device, its length should be equal to the conversion length $L_{C}$.

In the proposed structure, the silicon wire height $h_{s}$ and width $w_{s}$ are equal to $380 \mathrm{~nm}$, while the width of the $\mathrm{SiO}_{2}$ base strip is taken as $w_{b}=50$ $\mathrm{nm}$. The refractive index of the silicon and $\mathrm{SiO}_{2}$ are taken as 3.48 and 1.46 , respectively. In addition, $n_{o}$ and $n_{e}$ of the E7 material are fixed to 1.5024 and 1.6970 , respectively, at the operating wavelength $\lambda=1.55 \mu \mathrm{m}$ and at temperature of $25^{\circ} \mathrm{C}$. Meanwhile, the rotation angle $\varphi$ of the NLC is chosen as $45^{\circ}$.

Obtaining highly hybridness waveguides that can support the quasi-TE and quasi-TM modes, with almost equal amplitudes of the field components is the key factor in designing an efficient PR. In this study, the model hybridness is defined as (23)

$$
\text { Hybridness }=\max \left|H_{u}\right| / \max \left|H_{V}\right|
$$

where $u$ and $v$ are $x$ and $y$ for the quasi-TE mode while $y$ and $x$ for the quasi-TM mode, respectively.

The FVFDM (21) is used to obtain the model solution of the input and output waveguides shown in Fig. 1. The dominant $H_{y}$ and nondominant $H_{x}$ field profiles of the quasi-TE mode are shown in Fig. 2(a). It may be seen from this figure that the field profiles of the dominant $H_{y}$ and nondominant $H_{x}$ of the quasi-TE mode are quite different in shape with unequal amplitudes with low hybridness of 0.1 . Therefore, only a small amount of mode conversion can take place in such waveguides which can be used as input and output waveguides. On the other hand, the dominant $H_{y}$ and nondominant $H_{x}$ field profiles of inset shows the director of the NLC with a rotation angle $\varphi$.

the quasi-TE mode of the suggested PR are shown in Fig. 2(b). It can be noticed that the field profiles of the dominant $H_{y}$ and nondominant $H_{x}$ components of the quasi-TE mode are very similar with high model hybridness of 0.9915 . This means that the proposed PR supports highly hybrid modes which can be used for designing an efficient PR.

The modal hybridness can be mainly affected by the rotation angle of the NLC and the structure geometrical dimensions. The effect of the rotation angle on the modal hybridness and conversion length is first studied. In this investigation, the silicon wire height $h_{s}$ and width $w_{\mathrm{s}}$, and the width of the $\mathrm{SiO}_{2}$ base strip $w_{b}$ are fixed to $380 \mathrm{~nm}, 380$ $\mathrm{nm}$, and $50 \mathrm{~nm}$, respectively. Fig. 3 shows the variation of the conversion length and modal hybridness of the quasi-TE and TM modes, with the rotation angle $\varphi$. It is evident from this figure that the hybridness has its maximum value of 0.99155 and 0.97434 for the quasi-TE and TM modes, respectively, at rotation angle $\varphi=45^{\circ}$. Therefore, complete polarization conversion can take place at $\varphi=45^{\circ}$. However, if $\varphi$ is deviated from $45^{\circ}$, the modal hybridness is reduced to reach its minimum values at $\varphi=0^{\circ}$ and $90^{\circ}$. Moreover, Fig. 3 reveals that the rotation angle can affect the conversion length of the proposed PR. The conversion length decreases from 27.94 to $11.67 \mu \mathrm{m}$ by increasing the rotation angle from $0^{\circ}$ to $45^{\circ}$. If $\varphi$ is further increased to $90^{\circ}$ the conversion length will be increased to $28.64 \mu \mathrm{m}$.

Delta University for Science and Technology

Coastal International Road, Mansoura, Gamasa City, Dakahlia, Egypt

E-mail: dusj@deltauniv.edu.eg

Journal homepage: www.deltauniv.edu.eg/dusj 

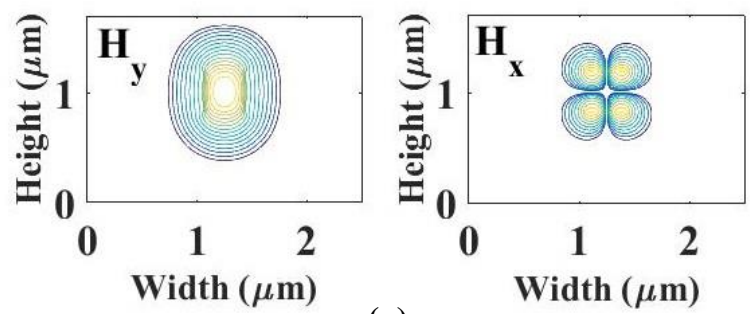

(a)
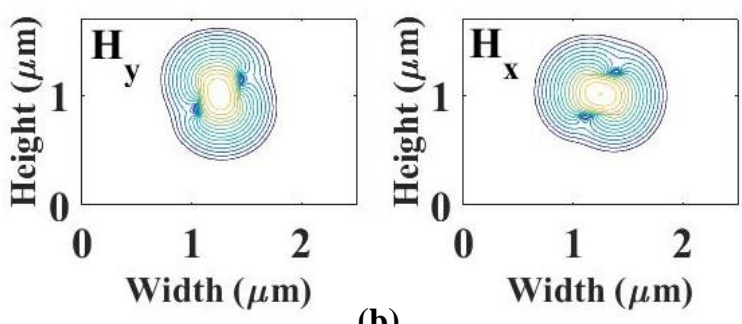

(b)

Fig. 2. Contour plots of the dominant $\mathrm{H}_{y}$ and nondominant $\mathrm{H}_{x}$ field profiles of the fundamental quasi-TE modes for (a) the input/output waveguides and (b) the proposed PR waveguide.

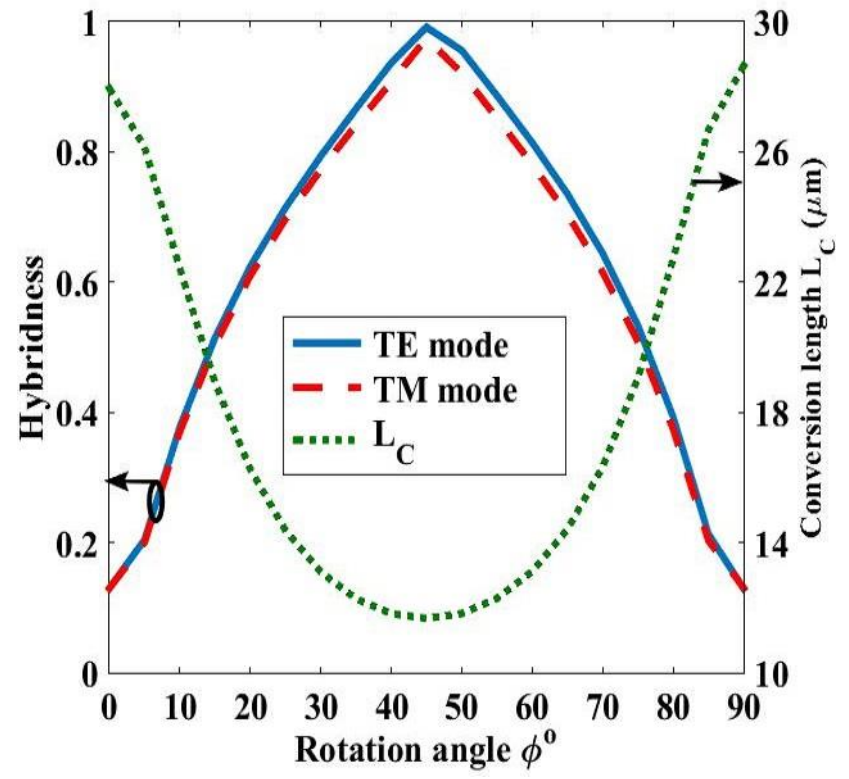

Fig. 3. Variation of the modal hybridness and conversion length with the rotation angle $\varphi$ of the director of the NLC.

The contour plots of the field components $\mathrm{H}_{\mathrm{x}}$ and $\mathrm{H}_{\mathrm{y}}$, calculated using the FVFD-BPM (17), of the input TE mode at different propagation distances $\mathrm{z}$ $=0, L_{\pi} / 2, L_{\pi}$ are shown in Fig. 4. It may be seen from this figure that as the TE input mode is launched from the input waveguide into the PR waveguide, the input power excites the hybrid modes along the PR waveguide where the dominant component $\mathrm{H}_{\mathrm{y}}$ is gradually decay while the nondominant component $\mathrm{H}_{\mathrm{x}}$ is gradually reinforced, till a relatively complete polarization conversion from the TE mode to the TM mode is occurred at $\mathrm{z}=L_{\pi}$ which is equal to $11.67 \mu \mathrm{m}$.
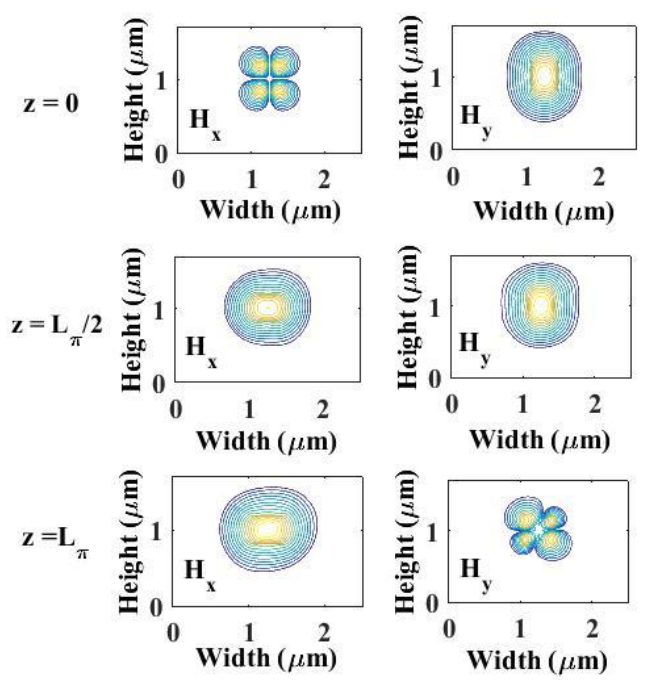

Fig. 4. Contour plot of $\mathrm{H}_{\mathrm{x}}$ and $\mathrm{H}_{\mathrm{y}}$ field components at z=0, $L_{\pi} / 2$, and $L_{\pi}$.

The effect of the silicon wire height $h_{s}$ on the modal hybridness is also studied. In this investigation, the silicon wire width $w_{\mathrm{s}}$, and the width of the $\mathrm{SiO}_{2}$ base strip $w_{b}$ are taken as 380 $\mathrm{nm}$, and $50 \mathrm{~nm}$, respectively. However, the rotation angle $\varphi$ is set to $45^{\circ}$. Fig. 5 shows the variation of the hybridness of the quasi-TE and TM modes with the silicon wire height. It is evident from this figure that the hybridness increases with increasing the height of the silicon wire until the hybridness reaches its maximum value for the quasi-TE and TM modes at $h_{s}=380$ $\mathrm{nm}$. However, if $h_{s}$ is further increased, the hybridness is reduced again.

Next, the effect of the silicon wire width $w_{s}$ on the modal hybridness is studied while the silicon wire height $h_{\mathrm{s}}$, the width of the $\mathrm{SiO}_{2}$ base strip $w_{b}$, and the rotation angle $\varphi$ are taken as $380 \mathrm{~nm}, 50 \mathrm{~nm}$, and $45^{\circ}$ respectively. Fig. 6 shows the variation of the hybridness of the quasi-TE and TM modes with the silicon wire width. It can be noticed from this figure that as the width of the silicon wire

Delta University for Science and Technology

Coastal International Road, Mansoura, Gamasa City, Dakahlia, Egypt

E-mail: dusj@deltauniv.edu.eg

Journal homepage: www.deltauniv.edu.eg/dusj 
increases, the hybridness of the two modes increases until its maximum value at $\mathrm{w}_{s}=380 \mathrm{~nm}$. However, if $w_{s}$ is further increased, the hybridness is reduced from its minimum value.

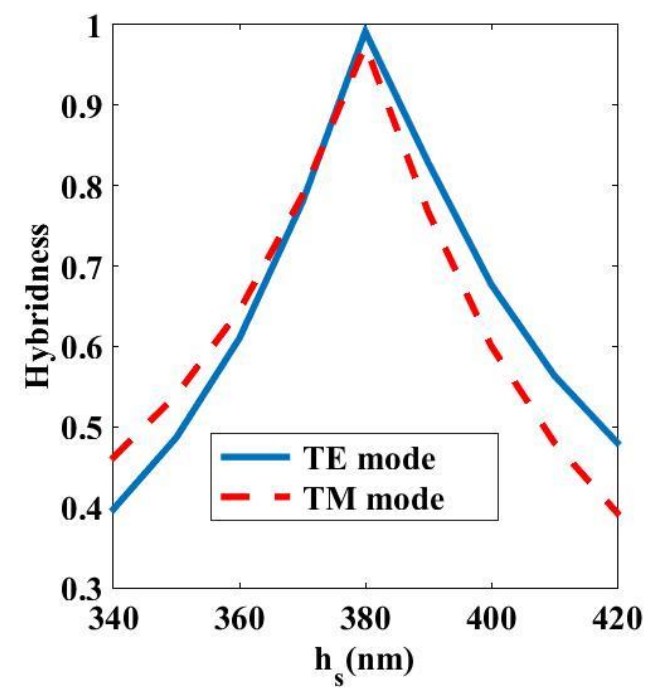

Fig. 5. Variation of the modal hybridness with the height of the silicon wire $h_{s}$.

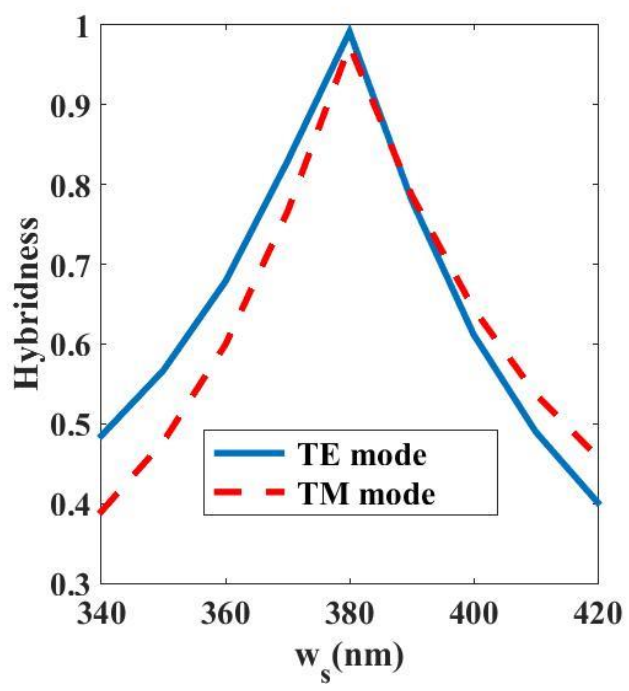

Fig. 6. Variation of the modal hybridness with the width of the silicon wire $w_{s}$.

The effect of the width of the $\mathrm{SiO}_{2}$ base strip $w_{b}$ is also reported. The numerical results reveal that the width of the $\mathrm{SiO}_{2}$ base strip has a slight effect on the modal hybridness and hence the conversion length and conversion ratio. The variations of the TM polarized powers $P_{x}$ for the TE excitation along the propagation direction at $w_{b}, 40 \mathrm{~nm}, 50$ $\mathrm{nm}$, and $60 \mathrm{~nm}$ are examined. In this analysis, the silicon wire height $h_{\mathrm{s}}$ and width $w_{s}$, and the rotation angle $\varphi$ are fixed as $380 \mathrm{~nm}, 380 \mathrm{~nm}$, and $45^{\circ}$ respectively. The maximum values of $P_{x}$ at $w_{b}$ $=40,50$, and $60 \mathrm{~nm}$ are found to be 0.9726 , 0.9727, and 0.9725, respectively. The corresponding conversion lengths are equal to $11.73,11.67$, and $11.8 \mu \mathrm{m}$, respectively. Therefore, the maximum $P_{x}$ and minimum $L_{C}$ are obtained at $w_{b}=50 \mathrm{~nm}$.

Maximizing the (ER), performance parameter, is also considered in designing the proposed PR, where the ER is defined as (8)

$$
E R=10 \log _{10}\left(\frac{P_{T M}}{P_{T E}}\right)
$$

where $P_{T E}$ and $P_{T M}$ are the respective output powers for the quasi-TE and quasi-TM modes at the output port for a quasi-TE input mode excitation, and a similar expression can be released for a quasi-TM input mode.

The reported PR with $h_{s}=380 \mathrm{~nm}, w_{s}=380 \mathrm{~nm}$, $w_{b}=50 \mathrm{~nm}$, and $\varphi=45^{\circ}$ offers a maximum hybridness of 0.9915 and a maximum ER of 15.5 $\mathrm{dB}$ with a device length of $11.67 \mu \mathrm{m}$. Therefore, such design will be considered as the appropriate device in the following simulations.

Studying the fabrication tolerances of the structural parameters and their effects on the polarization rotator performance has a great importance. The rotation angle of the director of the NLC is the first considered. Fig. 7 shows the variation of the ER values for TE to TM and TM to TE modes with the rotation angle $\varphi$ in the range from $25^{\circ}$ to $65^{\circ}$. It is found that the behavior of the ER variation with the rotation angle is very similar to the behavior of the modal hybridness, as shown in Fig. 3. It has been shown before that the maximum hybridness occurs at $\varphi=45^{\circ}$.

Delta University for Science and Technology

Coastal International Road, Mansoura, Gamasa City, Dakahlia, Egypt

E-mail: dusj@deltauniv.edu.eg

Journal homepage: www.deltauniv.edu.eg/dusj 


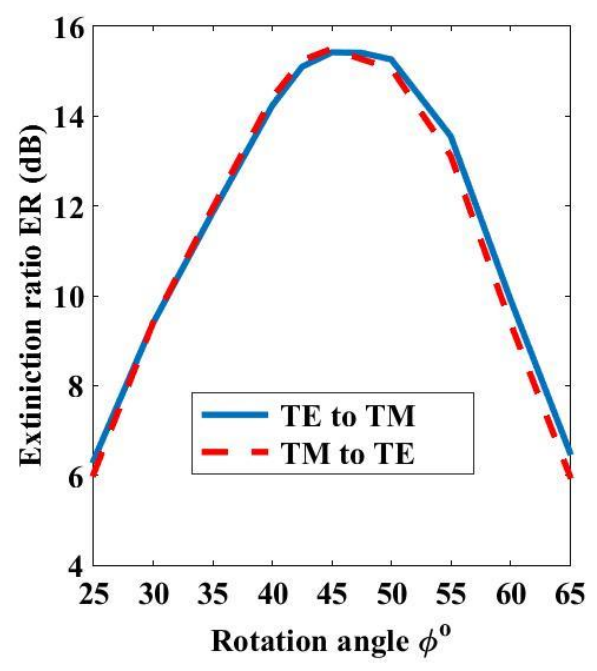

Fig. 7. Variation of the ER with the rotation angle of the director of the NLC $\varphi$.

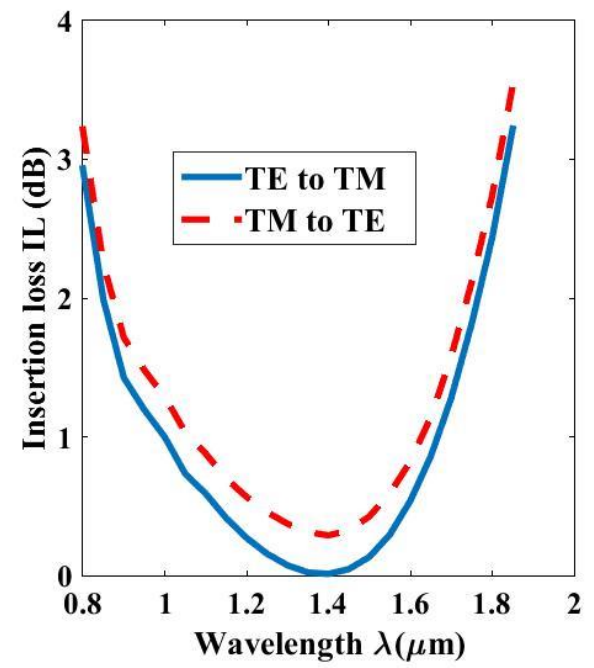

Fig. 8. Variation of the IL with the operating wavelength $\lambda$.

Similarly, the value of the ER is maximum at $\varphi$ $=45^{\circ}$ and any deviation from $\varphi=45^{\circ}$ results in reduction of the values of the hybridness and the ERs. It should be noted that when fabricating the device, to keep the maximum ER always better than $10 \mathrm{~dB}$, the rotation angle should be kept within the ranges from $30^{\circ}$ to $59^{\circ}$ and from $30^{\circ}$ to $58^{\circ}$ for $\mathrm{TE}$ to $\mathrm{TM}$ and for $\mathrm{TM}$ to $\mathrm{TE}$ modes, respectively, which are considered as a large fabrication tolerance for the suggested design. The fabrication tolerances and effects of the other structural geometrical parameters, such as $h_{s}, w_{s}$, and $w_{b}$ are also studied and summarized in table 1 .
Fig. 8 shows the variation of wavelength dependent IL for TE to TM and for TM to TE modes. From this figure, it may be seen that at the operating wavelength of $1.55 \mu \mathrm{m}$, the IL is equal to $0.29 \mathrm{~dB}$ for TE to TM mode and $0.59 \mathrm{~dB}$ for TM to TE mode. In addition, the IL values are less than $1 \mathrm{~dB}$ over a broad bandwidth of $725 \mathrm{~nm}$ and $560 \mathrm{~nm}$ for TE to TM and for TM to TE modes, respectively. The IL parameter is defined as (24).

$$
I L=-10 \log _{10}\left(\frac{P_{O M}}{P_{I N P U T}}\right)
$$

Table 1. Summary of the variation tolerances of the structural parameters $\varphi, h_{s}, w_{s}$, and $\lambda$ to keep the ER always better than $10 \mathrm{~dB}$.

\begin{tabular}{|c|c|c|}
\hline Parameter & $\begin{array}{c}\text { TE to TM } \\
\text { range }\end{array}$ & $\begin{array}{c}\text { TM to TE } \\
\text { range }\end{array}$ \\
\hline$\varphi$ & $30^{\circ}-59^{\circ}$ & $30^{\circ}-58^{\circ}$ \\
\hline$h_{s}$ & $371-392 \mathrm{~nm}$ & $371-391 \mathrm{~nm}$ \\
\hline$w_{s}$ & $368-391 \mathrm{~nm}$ & $369-391 \mathrm{~nm}$ \\
\hline$\lambda$ & $\begin{array}{c}860-1780 \\
\mathrm{~nm}\end{array}$ & $\begin{array}{c}890-1790 \\
\mathrm{~nm}\end{array}$ \\
\hline
\end{tabular}

\section{Conclusions}

A compact PR based on SOI and NLC has been presented and analyzed using the methods of FVFDM and FVFD-BPM. The PR has a device length of $11.67 \mu \mathrm{m}$ with $15.5 \mathrm{~dB}$ ER for both polarization conversions at $1.55 \mu \mathrm{m}$ wavelength. The IL is equal to $0.29 \mathrm{~dB}$ and $0.59 \mathrm{~dB}$ for TE to $\mathrm{TM}$ and for TM to TE conversions, respectively. Moreover, it offers a broad bandwidth of (860 $1780 \mathrm{~nm})$ for TE to TM and $(890-1790 \mathrm{~nm})$ for TM to TE to keep the ER better than $10 \mathrm{~dB}$. In addition, it is easy for fabrication and has a stable performance with variations in the operating wavelength and fabrication errors. The results shown here demonstrate that proposed PR can be employed to achieve strong polarization conversion in many applications.

\section{References}

(1) M. Paniccia and N. Photonics, "Integrating silicon photonics," Nat. Photon., vol. 4, no. 8, pp. 498-499, Aug. 2010.

Delta University for Science and Technology

Coastal International Road, Mansoura, Gamasa City, Dakahlia, Egypt

E-mail: dusj@deltauniv.edu.eg

Journal homepage: www.deltauniv.edu.eg/dusj 
(2) W. N. Ye and Y. Xiong, "Review of silicon photonics: history and recent advances," J. Modern Opt., vol. 60, no. 16, pp. 1299-1320, Oct. 2013.

(3) T. Barwicz, M. R. Watts, M. A. Popović, P. T. Rakich, L. Socci, F. X. Kärtner, E. P. Ippen, and H. I. Smith, "Polarization transparent microphotonic devices in the strong confinement limit," Nat. Photon., vol. 1, no. 1, pp. 57-60, Jan. 2007.

(4) L. Liu, Y. Ding, K. Yvind, and J. M. Hvam, "Silicon-on-insulator polarization splitting and rotating device for polarization diversity circuits," Opt. Express, vol. 19, no. 13, pp. 12646-12651, Jun. 2011.

(5) Y. Sun, Y. Xiong, and W. N. Ye, "Compact SOI Polarization Rotator Using Asymmetric Periodic Loaded Waveguides," IEEE Photon. J., vol. 8, no. 1, pp. 1-8, Feb. 2016.

(6) H. Deng, D. O. Yevick, C. Brooks, and P. E. Jessop, "Design rules for slantedangle polarization rotators," J. Lightw. Technol., vol. 23, no. 1, pp. 432-445, Jan. 2005.

(7) C. Brooks, P. E. Jessop, H. Deng, D. O. Yevick, and G. Tarr, "Passive siliconon-insulator polarization-rotating waveguides," Opt. Eng., vol. 45, no. 4, pp. 044603-1-044603-5, Apr. 2006.

(8) H. Deng, D. O. Yevick, C. Brooks, and P. E. Jessop, "Fabrication tolerance of asymmetric silicon-on-insulator polarization rotators," J. Opt. Soc. Am. $A$, vol. 23, no. 7, pp. 1741-1745, Jul. 2006.

(9) Z. Wang, D. Dai, "Ultrasmall Sinanowire-based polarization rotator," $J$. Opt. Soc. Am. B, vol. 25, no. 5, pp. 747753, May 2008.

(10) A. Barh, B. M. A. Rahman, R. K. Varshney, and B. P. Pal, "Design and performance study of a compact SOI polarization rotator at $1.55 \mu \mathrm{m}, " J$. Lightw. Technol., vol. 31, no. 23, pp. 3687-3693, Dec. 2013.

(11) S. Soudi, and B. M. A. Rahman, "Design of compact polarization rotator using simple silicon nanowires," Appl. Opt., vol. 53, no. 34, pp. 8071-8077, Dec. 2014.

(12) J. Zhang, S. Zhu, H. Zhang, S. Chen, G. Qiang Lo, and D. L. Kwong, "An Ultracompact Surface Plasmon Polariton-Effect-Based Polarization Rotator," IEEE Photon. Technol. Lett., vol. 23, no. 21, pp. 1606-1608, Nov. 2011.

(13) M. Komatsu, K. Saitoh, and M. Koshiba, "Compact polarization rotator based on surface plasmon polariton with low insertion loss," IEEE Photon. J., vol. 4, no. 3, pp. 707-714, Jun. 2012.

(14) L. Jin, Q. Chen, and L. Wen, "Modecoupling polarization rotator based on plasmonic waveguide," Opt. Lett., vol. 39, no. 9, pp. 2798-2801, May 2014.

(15) P. Yeh and C. Gu, "Optics of Liquid Crystal Displays," Wiley, 1999.

(16) A. B. Fallahkhair, K. S. Li, and T. E. Murphy, "Vector finite difference modesolver for anisotropic dielectric waveguides," J. Lightw. Technol., vol. 26, no. 11, pp. 1423-1431, Jun. 2008.

(17) W. P. Huang and C. L. Xu, "Simulation of three-dimensional optical waveguides by a full-vector beam propagation method," IEEE J. Quantum Electron., vol. 29, no. 10, pp. 2639-2649, Oct. 1993.

(18) W. C. Chew, J. M. Jin, and E. Michielssen, "Complex coordinate stretching as a generalized absorbing boundary condition," Microw. Opt. Technol. Lett., vol. 15, no. 6, pp. 363369, Aug. 1997.

(19) S. S. A. Obayya, B. M. A. Rahman, and H. A. El Mikati, "New full vectorial numerically efficient propagation algorithm based on the finite element method," J. Lightw. Technol., vol. 18, no. 3, pp. 409-415, Mar. 2000.

(20) M. F. O. Hameed and S. S. A. Obayya, "Analysis of polarization rotator based on nematic liquid crystal photonic crystal fiber," J. Lightw. Technol., vol. 28, no. 5, pp. 806-815, Mar. 2010.

(21) J. Li, S. T. Wu, S. Brugioni, R. Meucci, and S. Faetti, "Infrared refractive

Delta University for Science and Technology

Coastal International Road, Mansoura, Gamasa City, Dakahlia, Egypt

E-mail: dusj@deltauniv.edu.eg

Journal homepage: www.deltauniv.edu.eg/dusi 
indices of liquid crystals," J. Appl. Phys., vol. 97, no. 7, pp. 073501-073501-5, Mar. 2005.

(22) G. Ren, P. Shum, X. Yu, J. Hu, G. Wang, and $Y$. Gong, "Polarization dependent guiding in liquid crystal filled photonic crystal fibers," Opt. Commun., vol. 281, no. 6, pp. 1598-1606, 2008.

(23) M. F. O. Hameed and S. S. A. Obayya, "Polarization Rotator Based on Soft Glass Photonic Crystal Fiber with Liquid Crystal Core," J. Lightw. Technol., vol. 29, no. 18, pp. 2725-2731, Sep. 2011.

(24) X. Wang, and J. Dong, "Ultrashort polarization rotator based on crosssymmetry waveguide," Opt. Commun., vol. 367, pp. 68-71, May 2016. 